\title{
The prognostic accuracy evaluation of SAPS 3, SOFA and APACHE II scores for mortality prediction in the surgical ICU: an external validation study and decision-making analysis
}

Antônio Luis Eiras Falcão ${ }^{1 *}$, Alexandre Guimarães de Almeida Barros ${ }^{1}$, Angela Alcântara Magnani Bezerra ${ }^{1}$, Natália Lopes Ferreira', Claudinéia Muterle Logato', Filipa Pais Silva², Ana Beatriz Francioso Oliveira do Monte', Rodrigo Marques Tonella', Luciana Castilho de Figueiredo ${ }^{1}$, Rui Moreno², Desanka Dragosavac ${ }^{1}$ and Nelson Adami Andreollo'

\begin{abstract}
Background: The early postoperative period is critical for surgical patients. SOFA, SAPS 3 and APACHE II are prognostic scores widely used to predict mortality in ICU patients. This study aimed to evaluate these index tests for their prognostic accuracy for intra-ICU and in-hospital mortalities as target conditions in patients admitted to ICU after urgent or elective surgeries and to test whether they aid in decision-making. The process comprised the assessment of discrimination through analysis of the areas under the receiver operating characteristic curves and calibration of the prognostic models for the target conditions. After, the clinical relevance of applying them was evaluated through the measurement of the net benefit of their use in the clinical decision.

Results: Index tests were found to discriminate regular for both target conditions with a poor calibration (C statistics-intra-ICU mortality AUROCs: APACHE II 0.808, SAPS 30.821 and SOFA 0.797/in-hospital mortality AUROCs: APACHE II 0.772, SAPS 30.790 and SOFA 0.742). Calibration assessment revealed a weak correlation between the observed and expected number of cases in several thresholds of risk, calculated by each model, for both tested outcomes. The net benefit analysis showed that all score's aggregate value in the clinical decision when the calculated probabilities of death ranged between 10 and $40 \%$.
\end{abstract}

Conclusions: In this study, we observed that the tested ICU prognostic scores are fair tools for intra-ICU and in-hospital mortality prediction in a cohort of postoperative surgical patients. Also, they may have some potential to be used as ancillary data to support decision-making by physicians and families regarding the level of therapeutic investment and palliative care.

Keywords: Prognostic scores, Critical care, Surgical intensive care unit

\footnotetext{
*Correspondence: aefalcao@gmail.com

${ }^{1}$ Intensive Care Unit, Discipline of Physiology and Surgical Metabology,

Department of Surgery, Faculty of Medical Sciences, State University

of Campinas (Unicamp), Tessália Viera de Camargo St. 126, University

Town Zeferino Vaz, Campinas, São Paulo 13083-887, Brazil

Full list of author information is available at the end of the article
}

Springer Open

C The Author(s) 2019. This article is distributed under the terms of the Creative Commons Attribution 4.0 International License (http://creativecommons.org/licenses/by/4.0/), which permits unrestricted use, distribution, and reproduction in any medium, provided you give appropriate credit to the original author(s) and the source, provide a link to the Creative Commons license, and indicate if changes were made. 


\section{Background}

Surgical procedures continue to evolve, and patients with advanced age, frailty, and comorbidities are exposed to interventions with different levels of invasiveness, complexity, morbidity, and mortality-proposed classification systems grade complications from those procedures as simple symptomatic situations to conditions requiring surgical, endoscopic or radiological reintervention and life-threatening organ failure [1, 2]. Therefore, admission to ICU for postoperative recovery is common for surgical patients $[1,2]$. Nevertheless, admission to ICU is associated with potentially harmful situations like invasive monitoring and painful procedures [3]. Thus, a precise evaluation of the initial clinical condition, the type of procedure, and the final operative status is necessary to inform patients and physicians about the risk of complications and poor outcomes and to aid tailoring proportional therapeutic efforts.

Among many proposed prediction scores, Sequential Organ Failure Assessment (SOFA), Simplified Acute Physiology Score 3 (SAPS 3) and Acute Physiology and Chronic Health Disease Classification System II (APACHE II) are prognostic models that use clinical and laboratory variables to predict in-hospital mortality [48]. APACHE II and SAPS 3 were derived from a cohort of general ICU patients, while a consensus panel proposed SOFA as an organ dysfunction measurement score. Their performance was extensively assessed in several populational subgroups including mixed surgical-medical patients, post-cardiovascular surgical patients, and oncologic patients with heterogeneous results [9-12]. Therefore, external validation remains essential to evaluate the accuracy of them in new population subgroups and in different settings of care over time.

Moreover, traditional statistical methods use metrics based on sensitivity and specificity to assess prediction model's accuracy. However, the relationship between the measurement of accuracy and its clinical usefulness is a gray zone $[13,14]$. The decision analysis approach is an alternative to evaluate the clinical significance of applying those models and provides information into the clinical consequences of using them $[13,14]$. This strategy has been used to test for the net benefit of using SAPS II to end-of-life care decisions and to evaluate the net benefit of a new model based on CURB-65 and C-reactive protein to guide decision-making in ICU-admitted patients with success $[15,16]$.

This study aimed to validate and compare the performance of SOFA, SAPS 3 and APACHE II for intra-ICU and in-hospital mortalities as the target conditions in a cohort of mixed surgical patients admitted to ICU for postoperative recovery and to test whether they aid in the clinical decision-making.

\section{Methods}

This study was a prospectively defined analysis of a registry-based data validation cohort, gathered from consecutively admitted patients to a surgical ICU of a tertiary university hospital in Brazil, from January 1, 2013, to December 31, 2016. Our electronic database is continuously fed with predefined clinical and laboratory information from every patient admitted to our surgical ICU. Patients were followed daily during their ICU stay and then tracked for their final hospital status as discharged or deceased. The target condition of interest was the death of any cause in ICU or hospital. Variables, coefficients, and equations used for the index tests (SOFA, APACHE II, and SAPS 3) calculations were based on original publications without any adjustment or updating and are available upon request [4-6, 8]. APACHE II, SAPS 3 and SOFA scores were calculated after the first day of ICU admission using data collected at the prespecified time frame. This study was a registry-based data analysis with outcomes and predictors available before the beginning of any form of statistical analysis. Therefore, the blindness of outcomes or predictors was not employed. We followed the standards for reporting diagnostic accuracy (STARD) statement and the transparent reporting of a multivariable prediction model for individual prognosis or diagnosis (TRIPOD) statement recommendations for validation studies (Additional file 1: Figure S1) $[17,18]$.

We did not perform any formal statistical method for sample size calculation and evaluated all patients available in our database for enrollment. However, considering that more than 100 events were observed for intra-ICU mortality and more than 250 events for in-hospital mortality, we believe that our sample size is satisfactory.

Patients eligibility criteria for study enrollment were age 18 or above and admission to surgical ICU for postoperative recovery of an elective or urgent surgical procedure. Patient data were excluded only if the target condition information was missing. Noteworthy, there were no patient's exclusions after application of eligibility criteria. Our eligibility criteria were restrictive, allowing only surgical patients enrollment. These criteria contrast with original development cohorts of SAPS 3 and APACHE II. The SAPS 3 cohort included the first ICU admission of patients aged 16 or more and excluded data from patients lacking information about any admission or discharge variables. The APACHE II cohort consecutively included ICU-admitted patients for a medical or surgical reason and excluded patients that were missing any admission variable information or submitted to a coronary artery bypass graft surgery. These inclusion criteria are in contrast with our sample that enrolled patients submitted to any surgical 
procedure and enrolled those who had admission data missing. We handled missing values in predictor variables with multiple imputations. This procedure was performed with SPSS version 22 using a linear regression model. The variables included in the multiple imputation model were intra-ICU and in-hospital mortalities, age, sex, type of surgery, SAPS 3, APACHE II, and SOFA scores. Ten imputed datasets were created, and areas under the receiver operating characteristic curve had their sensitivities and specificities averaged to generate the final curve used in our results.

Our ICU provides a mixed model of care with full-time intensivists, nurses, assistants, respiratory therapists, dietitians, and attending physicians. A minimum standardized level of care was provided, consisting of a daily checklist called ABCD-preV (Additional file 2: Table S1) [19], in order to minimize therapeutic variations inside the population that could change the probability of the outcome and biased the results.

We evaluated the predictive performance of the index tests in a cohort of general surgical patients by estimating their discrimination and calibration. Discrimination reflects the capacity of a prediction model to differentiate between those who do and do not develop the defined target condition during the study period. For the measurement of discrimination, we used the concordance index (C-index) statistic through the calculation of the area under the receiver operating characteristic curve (AUROC) with intra-ICU or in-hospital mortality as the binary endpoints. A value of 0.5 for AUROC signifies chance and means that the predictor in analysis cannot distinguish between a positive or an adverse outcome while a value of 1 represents perfect discrimination. Discrimination was classified according to AUROC values as follows: $0.90-1$ excellent, $0.80-0.90$ good, $0.70-0.80$ fair, $0.60-0.70$ poor and $0.50-0.60$ fail [20]. The DeLong method was used to compare whether differences between different models AUROC's were statistically significant [21]. Calibration reflects how well intra-ICU and in-hospital mortalities predicted by each model agree with the observed outcomes. This relation was shown graphically by clustering patients in tenths of predicted risk according to each model and plotting the expected against the observed number of cases. A smoothed line was drawn over the entire predicted probability range to augment the observed correlation. A well-calibrated model predicts over a line slope around $45^{\circ}$. The calibration plot also indicates the magnitude and direction of the model's miscalibrations. For statistical analysis of the model's predictive performance, we employed the Hosmer-Lemeshow goodness-of-fit test [22]. In an adequate sample size, results with $p$ values higher than 0.05 indicate a good agreement between the model's predicted probabilities and observed outcome rates.

Median follow-up was calculated for intra-ICU and inhospital periods according to the reverse Kaplan-Meier survival function that uses the event indicator reversed and censoring becomes the outcome of interest.

A decision curve analysis was developed to describe and compare the clinical utility of tested models. Logistic regression was used to convert the model's calculated values into predicted probabilities of death. Patients were defined as high risk if their intra-ICU or in-hospital mortality probabilities were higher than the prognostic model set probability threshold. Net benefit for different threshold values of each model was calculated according to Vickers et al. and compared to the possible clinical strategy of considering that all patients were positive for the outcome and treated them all and that all patients were negative for the outcome and received no treatment [13, 14].

Statistical analyses were performed using MedCalc version 18 and SPSS version 22. Continuous variables were reported as a mean and standard deviation or median and interquartile ranges whether they follow a normal distribution or not. Categorical variables were presented as count and proportion. Univariate analysis was performed using appropriated tests for continuous and categorical variables to assess association with mortality. Relative risks for mortalities were calculated after adjustment for illness severity. This procedure was performed using a case-control matching strategy with severity scores (SOFA, SAPS 3, and APACHE II) as specific criteria. A two-tailed $p$ value of less than 0.05 was considered statistically significant.

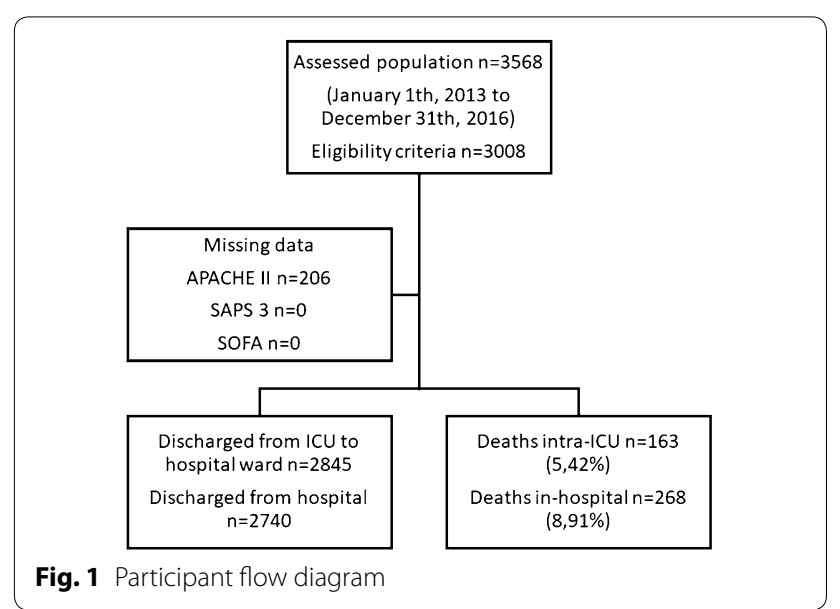




\section{Results}

We assessed an initial population of 3568 patients and polled out 3008 patients for further analysis according to our eligibility criteria (Fig. 1). The main reason for exclusion was ICU admission motivated by a medical reason not related to a surgical procedure. All patients assessed had their outcomes available, and no further exclusion was necessary. APACHE II, SAPS 3, and SOFA were calculated at appropriated timepoints and patients followed until they deceased or discharged from the hospital. APACHE II data were missing in 206 patients and had their values calculated using multiple imputations. Analyzed population demography and clinical features are summarized in Tables 1 and 2 and Additional file 3: Figure S2. In-hospital and intra-ICU mortality rates were $8.91 \%$ and $5.42 \%$, respectively, during the evaluated period. Median follow-up period was 12 days for in-hospital length of stay and three days for intra-ICU length of stay. Mechanical ventilation was associated with the highest relative risk for ICU mortality [RR 3.97 (95\% CI 1.59-9.95)].

C-index statistics were calculated for each prognostic model with intra-ICU and in-hospital mortalities as dependent target conditions (Table 3). The following AUROCs were obtained with intra-ICU mortality as the outcome: APACHE II 0.808 (95\% CI 0.794-0.822), SAPS 30.821 (95\% CI $0.807-0.835$ ), and SOFA 0.797 (95\% CI 0.783-0.812). Considering in-hospital mortality, the following AUROCs were observed: APACHE II 0.772 (95\% CI $0.757-0.787)$, SAPS 30.790 (95\% CI $0.775-$ 0.804), and SOFA 0.742 (95\% CI 0.726-0.758). Pairwise comparison among prognostic models resulted in no significant difference between them, except for SAPS 3 and SOFA score AUROCs difference that could not be explained by chance when in-hospital mortality was the target condition (Table 4; Fig. 2).

Table 1 Patient's baseline characteristics

\begin{tabular}{|c|c|c|c|c|c|c|c|c|c|}
\hline & \multirow[t]{2}{*}{ Total } & \multicolumn{4}{|l|}{ Intra-ICU } & \multicolumn{4}{|l|}{ In-hospital } \\
\hline & & Alive & Deaths & $p$ value & $\begin{array}{l}\text { Relative risk ( } 95 \% \\
\mathrm{CI})\end{array}$ & Alive & Deaths & $p$ value & $\begin{array}{l}\text { Relative risk (95\% } \\
\mathrm{Cl})\end{array}$ \\
\hline Age median (IQR) & $58(47-67)$ & $58(47-67)$ & $63(53-70)$ & $<0.001^{*}$ & & $57(46-67)$ & $63(54.5-71)$ & $<0.001^{*}$ & \\
\hline Male sex count (\%) & $1798(59.8)$ & 1693 & 105 & $0.21^{* *}$ & & 1631 & 167 & $0.37^{* *}$ & \\
\hline \multicolumn{10}{|c|}{ The urgency of the surgical procedure count (\%) } \\
\hline Urgent & $220(7.3)$ & 170 & 50 & & & 152 & 68 & & \\
\hline Elective & $2788(92.7)$ & 2675 & 113 & & & 2588 & 200 & & \\
\hline \multicolumn{10}{|c|}{ Preexistent conditions count (\%) } \\
\hline $\begin{array}{l}\text { Arterial hyperten- } \\
\text { sion }\end{array}$ & $1537(51.1)$ & 1452 & 85 & $0.72^{* *}$ & & 1394 & 143 & $0.40^{* *}$ & \\
\hline Diabetes mellitus & $634(21.1)$ & 604 & 30 & $0.39^{* *}$ & & 570 & 64 & $0.24^{* *}$ & \\
\hline Alcohol use & $371(12.3)$ & 347 & 24 & $0.34^{* *}$ & & 335 & 36 & $0.57^{* *}$ & \\
\hline Tobacco use & $1085(36.1)$ & 1029 & 56 & $0.64^{* *}$ & & 1001 & 84 & $0.09 * *$ & \\
\hline $\begin{array}{l}\text { Intra-ICU length of } \\
\text { stay days median } \\
\text { (IQR) }\end{array}$ & $3(2-5)$ & $3(2-5)$ & $7(3-15)$ & $<0.001^{*}$ & & & & & \\
\hline $\begin{array}{l}\text { In-hospital length of } \\
\text { stay days median } \\
\text { (IQR) }\end{array}$ & $12(8-20)$ & & & & & $11(7-19)$ & $17(9-34.5)$ & $<0.001^{*}$ & \\
\hline \multicolumn{10}{|c|}{ Severity Scores median (IQR) } \\
\hline SOFA & $3(2-6)$ & $3(2-6)$ & $7(5-9)$ & $<0.001^{*}$ & & $3(2-6)$ & $6(4-9)$ & $<0.001^{*}$ & \\
\hline APACHE $\|$ & $12(9-15)$ & $11(8-14)$ & $17(13-22)$ & $<0.001^{*}$ & & $11(8-14)$ & $16(13-20)$ & $<0.001^{*}$ & \\
\hline SAPS 3 & $36(28-44)$ & $36(28-43)$ & $52(43-60)$ & $<0.001^{*}$ & & $35(28-43)$ & $48(41-58)$ & $<0.001^{*}$ & \\
\hline \multicolumn{10}{|l|}{ Life support therapies } \\
\hline $\begin{array}{l}\text { Mechanical ventila- } \\
\text { tion count }(\%)\end{array}$ & 1491 (49.6) & 1333 & 158 & $<0.01^{* *}$ & 3.97 (1.59-9.95) & 1269 & 222 & $<0.01^{* *}$ & $1.44(1.07-1.93)$ \\
\hline $\begin{array}{l}\text { Length of mechani- } \\
\text { cal ventilation } \\
\text { days median (IQR) }\end{array}$ & $1(1-2)$ & $1(1-1)$ & $7(2-12)$ & $<0.01^{*}$ & & $1(1-1)$ & $5(2-11)$ & $<0.01^{*}$ & \\
\hline $\begin{array}{l}\text { Renal replacement } \\
\text { therapy count (\%) }\end{array}$ & $143(4.8)$ & 93 & 50 & $<0.01^{* *}$ & $1.9(1.42-2.53)$ & 78 & 65 & $<0.01^{* *}$ & $1.78(1.43-2.22)$ \\
\hline
\end{tabular}

*Mann-Whitney

**Chi-squared 
Table 2 Type of surgery distribution across patients

\begin{tabular}{|c|c|c|}
\hline Surgical specialties & $\begin{array}{l}\text { Number } \\
\text { of cases } \\
\text { count }(n)\end{array}$ & Percent \\
\hline \multicolumn{3}{|l|}{ Head and neck surgery } \\
\hline Tumor & 38 & 1.26 \\
\hline Others & 14 & 0.47 \\
\hline \multicolumn{3}{|l|}{ Cardiac surgery } \\
\hline Coronary artery bypass graft & 339 & 11.27 \\
\hline Thoracic aortic aneurysm & 89 & 2.96 \\
\hline Cardiac transplant & 24 & 0.80 \\
\hline Valve replacement & 189 & 6.28 \\
\hline Others & 50 & 1.66 \\
\hline \multicolumn{3}{|l|}{ Surgery of esophagus and abdomen } \\
\hline Liver & 67 & 2.23 \\
\hline Liver transplant & 141 & 4.69 \\
\hline Biliary tract & 133 & 4.42 \\
\hline Esophagus and stomach & 177 & 5.88 \\
\hline Colon, rectum, and anus & 195 & 6.48 \\
\hline Others & 4 & 0.13 \\
\hline \multicolumn{3}{|l|}{ Neurosurgery } \\
\hline Aneurysm & 105 & 3.49 \\
\hline Epilepsy & 84 & 2.79 \\
\hline Tumor & 317 & 10.54 \\
\hline Spine & 109 & 3.62 \\
\hline Decompressive craniectomy & 23 & 0.76 \\
\hline Ventriculostomy & 23 & 0.76 \\
\hline Others & 60 & 1.99 \\
\hline \multicolumn{3}{|l|}{ Thoracic surgery } \\
\hline Tumor & 70 & 2.33 \\
\hline Other & 57 & 1.89 \\
\hline \multicolumn{3}{|l|}{ Urology } \\
\hline Kidney transplant & 123 & 4.09 \\
\hline Tumor & 167 & 5.55 \\
\hline Others & 48 & 1.60 \\
\hline \multicolumn{3}{|l|}{ Vascular surgery } \\
\hline Abdominal aortic aneurysm & 164 & 5.45 \\
\hline Endarterectomy & 88 & 2.93 \\
\hline Others & 95 & 3.16 \\
\hline Trauma, orthopedic, and ophthalmic surgeries & 15 & 0.50 \\
\hline Total & 3008 & 100 \\
\hline
\end{tabular}

Table 3 Severity score's area under the receiver operating characteristic (AUROC) curves for hospital and ICU mortalities as outcomes

\begin{tabular}{lll}
\hline Severity score & $\begin{array}{l}\text { AUROC-in-hospital } \\
\text { mortality } \mathbf{( 9 5 \% ~ C l )}\end{array}$ & $\begin{array}{l}\text { AUROC-intra-ICU } \\
\text { mortality } \mathbf{( 9 5 \% ~ C l )}\end{array}$ \\
\hline APACHE II & $0.772(0.757-0.787)$ & $0.808(0.794-0.822)$ \\
SAPS 3 & $0.790(0.775-0.804)$ & $0.821(0.807-0.835)$ \\
SOFA & $0.742(0.726-0.758)$ & $0.797(0.783-0.812)$ \\
\hline
\end{tabular}

Next, patients were divided into approximately ten similar groups of risk defined by increasing order of estimated risk according to each prognostic model and expected, and observed deaths were calculated in each group. Calibration graphs were built plotting the expected and observed values for each group and goodness-of-fit tested with the Hosmer-Lemeshow statistics (Fig. 3; Table 5). Also, the ratios of observed and expected number of deaths in each risk group were plotted to show the overall fit of the tested models (Fig. 3). In summary, models had a poor calibration in extremities of risk, overestimating and underestimating intra-ICU and in-hospital mortality, respectively. Based on the HosmerLemeshow goodness-of-fit test, APACHE II and SAPS 3 had $p$ values above 0.05 while SOFA score showed a $p$ value lower than 0.05 which indicates miscalibration for both outcomes.

Then, we calculated the intra-ICU and the in-hospital probability of death given by each prognostic model in ICU admission and plotted decision curves to determine how they aid in decision-making (Fig. 4). For both target conditions, the net benefit curves of the tested prognostic models were similar regardless of the selected threshold. Although SOFA, SAPS 3, and APACHE II showed diverse discrimination and calibration features, they showed a positive net benefit in the 10-40\% range of death probability. Above or below this range, the net benefit of using them is no better than not treat any patient or treat them all, respectively.

\section{Discussion}

In this external validation study, we sought to evaluate the performance of prognostic models to predict intraICU, and in-hospital mortalities in a cohort of surgical patients admitted in ICU for postoperative recovery and tested how it could help in decision-making. Multivariable prognostic models analyzed were employed identical to their original descriptions, without any adjustments in variables selection or weighting. SAPS 3 and APACHE II were initially developed to predict hospital mortality, while SOFA was initially proposed as a measurement of organic dysfunction and posteriorly validated for mortality prediction in different subgroups of patients $[4,5$, $8,23]$. In development studies, SAPS 3 and APACHE II scores showed AUROCs of 0.825 and 0.863 , respectively. In a recent review of prognostic scores performance in low and mid-income countries, discrimination of SAPS 3 and APACHE II evaluated through AUROCs ranged between 0.7 and 0.9 for intra-ICU and in-hospital mortalities as outcomes [24]. It is important to stress out that our sample was enrolled in a tertiary university hospital from a high-income region of Brazil and may have features different from low- and mid-income settings that 
Table 4 Pairwise comparison of prediction scores AUROC curves

\begin{tabular}{lllll}
\hline Severity score & $\begin{array}{l}\text { Difference between AUROCs } \\
\text { in-hospital mortality }(\mathbf{9 5 \%} \mathbf{C l})\end{array}$ & $\boldsymbol{p}$ value & $\begin{array}{l}\text { Difference between AUROCs intra-ICU } \\
\text { mortality } \mathbf{( 9 5 \% ~} \mathbf{~ C l})\end{array}$ \\
\hline APACHE II versus SOFA & $0.0296(-0.004$ to 0.063$)$ & 0.0840 & $0.0109(-0.027$ to 0.049$)$ \\
APACHE II versus SAPS 3 & $0.0177(-0.014$ to 0.049$)$ & 0.2686 & $0.0130(-0.024$ to 0.05$)$ & 0.5748 \\
SAPS 3 versus SOFA & $0.0474(0.013-0.082)$ & 0.0068 & $0.0263(-0.013$ to 0.061$)$ & 0.4973 \\
\hline
\end{tabular}
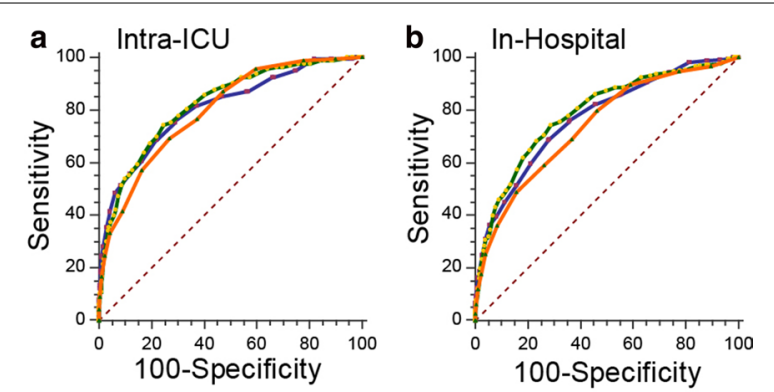

Fig. 2 Pairwise comparison of the prediction model's receiver operating characteristic (ROC) curves. ROC curves of different severity scores with intra-ICU (a) and in-hospital (b) mortality as the outcome. Green line-APACHE II; blue line-SAPS 3; orange line_-SOFA

may preclude extrapolation. To the best of our knowledge, none of the assessed prognostic models had their performance tested in a cohort exclusive of surgical patients from different specialties. Our data suggest fair to good discrimination of the tested models, with best results observed using SAPS 3 for prediction of both target conditions. APACHE II score was better calibrated for in-hospital mortality prediction than SAPS 3 and SOFA that trend to underestimate low-risk patient's and overestimate high-risk patient's probability of death. Scores prediction of intra-ICU mortality had a poor calibration with SAPS 3 fitting better among them.

In contrast to APACHE II and SAPS 3 that use features reflecting chronic conditions like the patient's age to estimate risk, SOFA measures six organic variables reflecting mostly acute conditions. In this study, our sample was composed mainly of patients admitted to elective surgical procedures with their baseline conditions optimized. Perhaps SOFA performed poorly because of the lack of correlation between its variables and the target conditions in our setting. It is possible that recalibration of SOFA's variables may improve its accuracy. Moreover, prognostic scores performance deteriorates over time and among different ICUs, especially calibration $[25,26]$. Therefore, it is critical to external validate prognostic scores overtime and before their utilization in new ICUs.

The traditional evaluation of prognostic scores using discrimination and calibration measurements is not new and conventional, and cannot define whether is worth using a particular model as an ancillary tool for decisionmaking or which of them is superior in practice $[13,20]$. We calculated the net benefit of tested models using different thresholds of the risk of death. Although death is a severe final event and false-negative and false-positive results limit the individual applicability of prognostic scores, the benefit of full therapeutic investment in certain patients admitted in ICU is unclear and may bring additional suffering and unnecessary resource utilization $[13,14]$. Our data suggest that APACHE II, SAPS 3, and SOFA calculated in admission may add information to help physicians and patients in decision-making about therapeutic management and palliative care when the calculated predicted risk of death is between 10 and $40 \%$ with no score superior to others. Although redundant in extremes of illness severity, mortality of patients with low and intermediate levels of risk is difficult to predict and gathering data from prognostic models may improve decisions about therapeutic management [13, 14, 27]. It is important to stress out that there was no observed net benefit to patients with high levels of risk for both target conditions. Maybe the small sample size in this subgroup of patients was insufficient to create a detectable signal by the tested prognostic models.

This study has several limitations that must be stressed out. Our cohort was derived from a single-center population with inclusion and exclusion criteria that yielded significant differences in demographic and clinical features compared with original multicentric cohorts used for SAPS 3 and APACHE II development $[4,5,8]$. SAPS 3 and APACHE II cohorts were composed of mixed clinical and surgical cases, with almost half of patients being unplanned admitted in ICU, which contrasts with our sample that was composed exclusively of surgical patients admitted to ICU for postoperative recovery mainly of elective surgeries. Patients were also iller in original SAPS 3 and APACHE II development cohorts as illustrated by the number of organic dysfunctions which was higher than in our cohort. For instance, the median SOFA in SAPS 3 original development cohort was 9 with an interquartile range of 6-11, while our patients had a median SOFA of 3 with an interquartile range of 2-6 [5, 8]. Although the length of ICU and hospital stay, age, and 
a

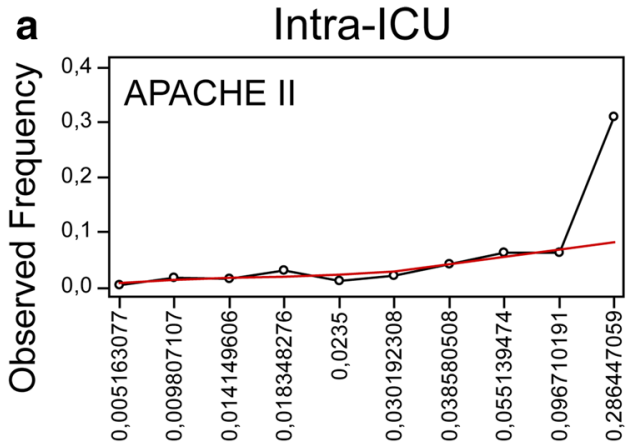

Expected Frequency

b

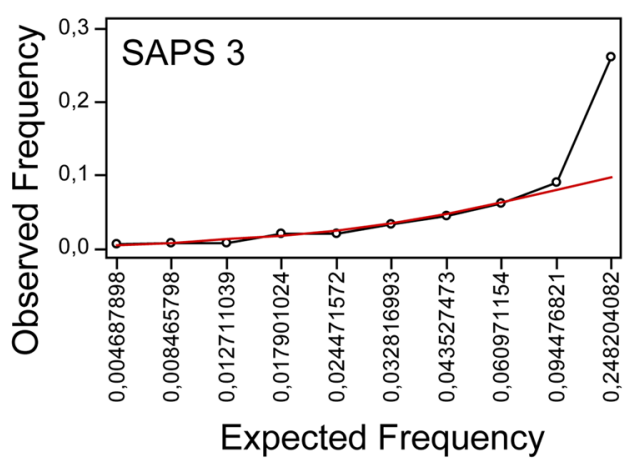

c

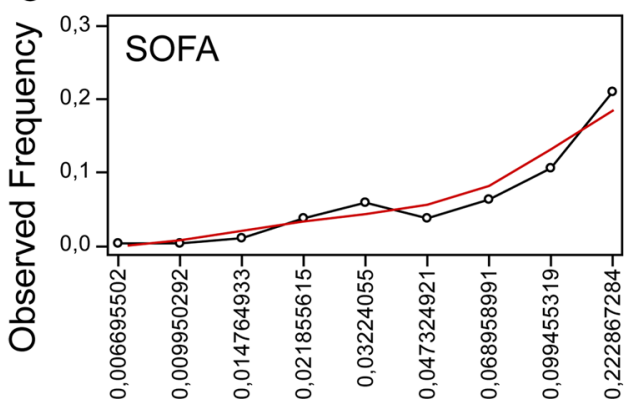

Expected Frequency

g

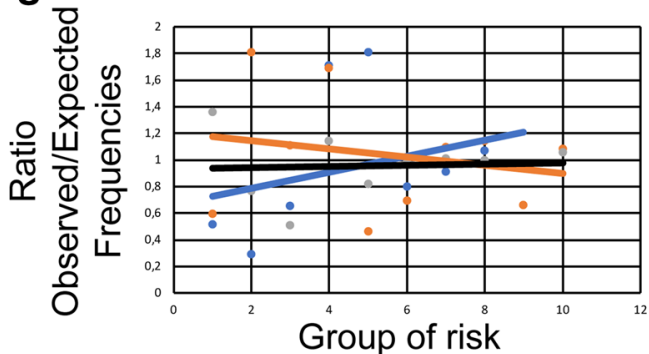

d

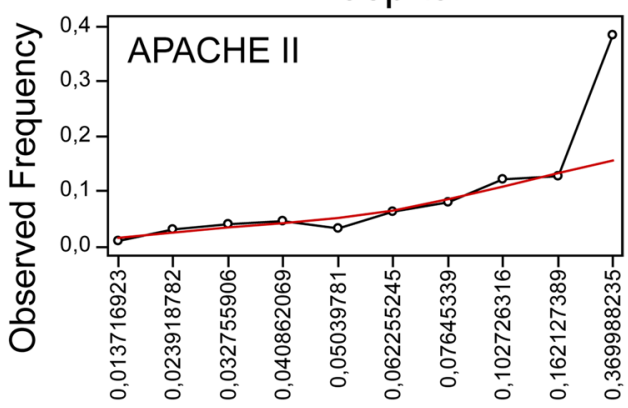

Expected Frequency

e

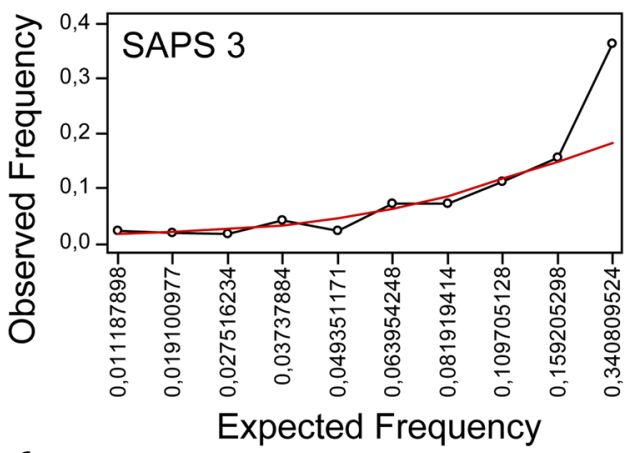

f

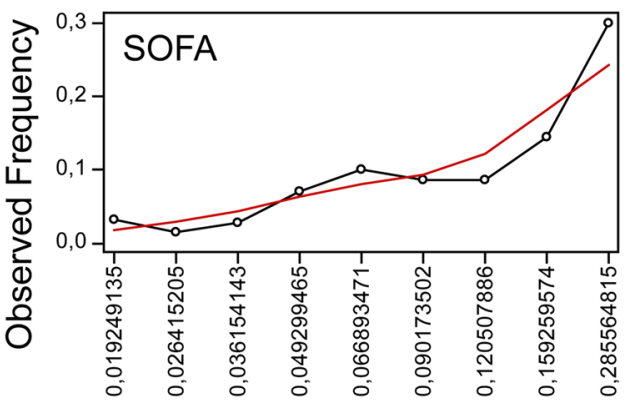

Expected Frequency

h

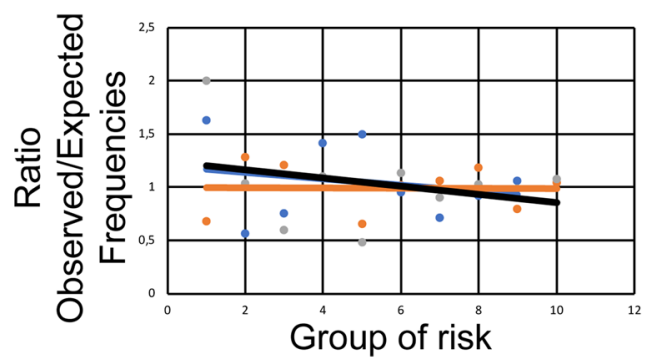

Fig. 3 Prediction models calibration plots. a-f Groups covering the entire predicted intra-ICU (a-c) or in-hospital (d-f) mortality probabilities calculated by each severity score (on the $x$-axis) plotted against observed frequencies (on the $y$-axis) (Dots linked by the black line). A LOWESS line (red), spanning $75 \%$ of local values, was created for each dataset to clarify the relationship between assessed variables and to shed light on the direction and magnitude of model miscalibration across the probability range. $\mathbf{g}, \mathbf{h}$ The ratios of observed over expected intra-ICU (g) or in-hospital (h) mortality probabilities, calculated by each prediction model (on the $y$-axis), were plotted against sequential clusters of risk (on the $x$-axis) to allow direct comparison between severity scores. Linear trend lines were created to aid in comparison. Orange line_APACHE Il; black line_-SAPS 3; blue line-SOFA 
Table 5 Prognostic model's calibration values for hospital and intra-ICU mortalities as outcomes

\begin{tabular}{llll}
\hline Severity score & $\begin{array}{l}\text { Hospital mortality } \\
\text { Hosmer and Lemeshow test-Chi- } \\
\text { squared (DF) }\end{array}$ & $\boldsymbol{p}$ value & $\begin{array}{l}\text { intra-ICU mortality } \\
\text { Hosmer and Lemeshow test-Chi- } \\
\text { squared (DF) }\end{array}$ \\
\hline SOFA admission & $18.04(7)$ & 0.0118 & $14.98(7)$ \\
SAPS 3 & $10.71(8)$ & 0.2189 & $2.02(8)$ \\
APACHE II & $7.89(8)$ & 0.4441 & $13.35(8)$ \\
\hline
\end{tabular}
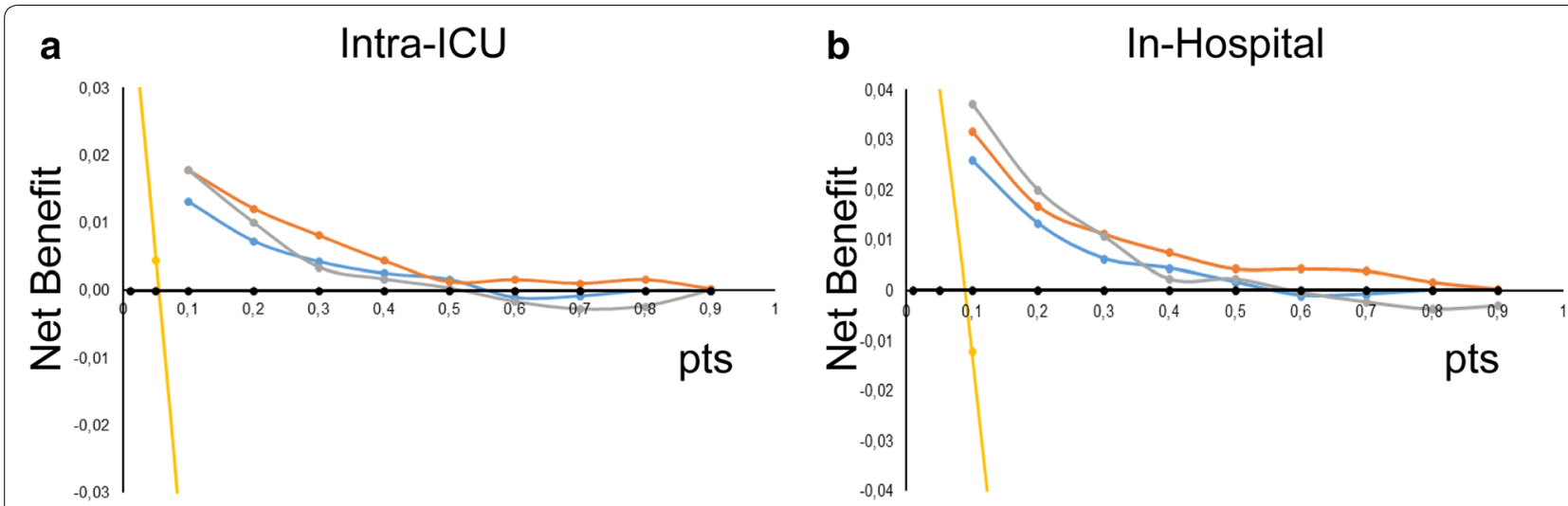

Fig. 4 Prediction models decision curves. a, b The net benefits of using each prediction model (on the $y$-axis) plotted for different thresholds of the probability of intra-ICU (a) or in-hospital (b) deaths (on the $x$-axis). The net benefit was calculated according to the following formula: net benefit $=[($ true-positive count $) / n]-[($ false-positive count $) / n] \times[\mathrm{pt} /(1-\mathrm{pt})]$ where $\mathrm{n}$ is the total number of patients and pt the threshold probability. Two lines representing the net benefit associated with the strategy of assuming all patients survived (no false positives) (black line) and that all patients died (yellow line) was drawn for comparison. Orange line_APACHE II; blue line—SOFA; gray line—SAPS 3

comorbidities profile were similar among our patients and original SAPS 3 and APACHE II cohorts, comparison of intra-ICU and in-hospital mortality reveals differences in outcome rates $[4,5,8]$. SAPS 3 and APACHE II original cohorts exhibited a broad spectrum of intra-ICU and in-hospital mortalities, with rates ranging between 10 and $30 \%$, while mortality rates observed in this study were both below $10 \%$. This difference may be in part explained by the features described above in the composition of analyzed cohorts, but also from selection and information bias, which are intrinsic to observational studies [18]. Also, it must be pointed out that the time difference between each cohort assembly creates a variance in features like therapeutic options available at the time that have a direct impact on analyzed outcomes. SAPS 3 database was built from data of patients admitted in ICUs of multiple countries from October to December 2002, while APACHE II database recruited patients between 1979 and 1982 in multiple ICUs from the USA $[4,5,8]$. It is in contrast with our database which collected data from patients admitted in one hospital ICU from 2013 to 2016. Differences in frequency of tested outcomes are an important feature that may impact the generalizability of results and conclusions of external validation studies. Comparison of the observed inhospital mortality rate in this study with those found in comparable cohorts showed similar frequencies [28-30]. Datasets from these studies were derived from elective and non-elective surgical patients in the postoperative period admitted in ICUs of European hospitals with similar features to the tertiary setting where our data were derived [28-30]. Correlation of our mortality frequencies with data from other Brazilian ICUs revealed similar in-hospital mortality although cohorts compositions were different [24, 31]. Another limitation was the small size of our cohort, especially in the high-risk subgroup of patients. This fact may account for part of the reasonable accuracy and poor calibration observed for the tested scores and the absence of net benefit to this subgroup of patients in decision-making.

\section{Conclusions}

In conclusion, this study assessed the performance of widely used prognostic scores for death prediction of surgical patients admitted in ICU for postoperative recovery. Observed results suggested that APACHE II, SAPS 3, and SOFA have regular discrimination features and poor calibration. Other studies showed similar results in different 
population subgroups, none using a cohort with characteristics of ours. Currently, prognostic scores are used for benchmarking, comparisons between ICUs performance and standardization of excellence. As previously suggested by others, our data support the fact that adopting those prognostic scores without further local external validation and adjustment may be misleading $[25,26]$.

Another point to be stressed out is that although the tested prognostic scores have a net benefit in death prediction of the low and intermediate level of risk surgical patients admitted in ICU, their performance was deficient when applied in the high level of risk patients which is the subgroup most susceptible to the futility of care. Therefore, before being ascribed as ancillary tools to aid in decision-making, improvements in the net benefit features generated using the tested prognostic models, especially in extremes of illness severity, must be sought. Noteworthy, no prognostic model should be used isolated to guide decision-making or replace clinical judgment. Further studies are needed to define the exact role the tested prognostic models may have as part of the decision-making process in ICU.

\section{Additional files}

\section{Additional file 1: Figure S1. STARD 2015 Checklist: Prediction Model}

Validation.

Additional file 2: Table S1. ABCD-preV checklist.

Additional file 3: Figure S2. Prediction scores distribution frequency. A-F-Patients distribution across severity scores values with intra-ICU $(\mathrm{A}, \mathrm{C}$ and $\mathrm{E})$ and in-hospital (B, D and F) mortality as outcomes. Blue bars represent survivors and green bars non-survivors.

\section{Abbreviations}

SOFA: Sequential Organ Failure Assessment; SAPS 3: Simplified Acute Physiology Score 3; APACHE II: Acute Physiology and Chronic Health Disease Classification System II; AUROC: area under the receiver operating characteristic curve; ICU: intensive care unit.

\section{Authors' contributions}

ALEF and AGAB conceived and designed the study, analyzed the data, and wrote the first and revised version of the manuscript. AAMB, MRB, and FPS contributed substantially reviewing data analysis and manuscript. ABFOM, RMT, and LCF contributed substantially with manuscript writing and revision. $\mathrm{RM}, \mathrm{DD}$, and NRA contributed with study design, manuscript writing, and revision. NLF contributed substantially with the writing of the revised version of this manuscript. All authors read and approved the final manuscript.

\section{Author details}

${ }^{1}$ Intensive Care Unit, Discipline of Physiology and Surgical Metabology, Department of Surgery, Faculty of Medical Sciences, State University of Campinas (Unicamp), Tessália Viera de Camargo St. 126, University Town Zeferino Vaz, Campinas, São Paulo 13083-887, Brazil. ${ }^{2}$ Unidade de Cuidados Intensivos Polivalente, Unidade de Cuidados Neurocríticos, Hospital de São José, Centro Hospitalar de Lisboa Central, Lisbon, Portugal.

\section{Acknowledgements}

We are thankful to all members of the intensive care unit of Unicamp's Teaching Hospital and Central Lisbon Hospital Center that contributed to this study.

\section{Competing interests}

The authors declare that they have no competing interests.

\section{Availability of data and materials}

The datasets used and analyzed during the current study are available from the corresponding author on reasonable request.

\section{Consent for publication}

Not applicable.

\section{Ethics approval and consent to participate}

The local ethics committee approved this study; Process No. CAAE 75821717.1.0000.5404. This study was observational, and every clinical decision was at the discretion of the attending physician. Therefore, informed consent was waived. The electronic database encrypted patient's identification and investigators had access only to relevant data for the study.

\section{Funding}

This study has not received any financial support from any source.

\section{Publisher's Note}

Springer Nature remains neutral with regard to jurisdictional claims in published maps and institutional affiliations.

Received: 28 August 2018 Accepted: 12 January 2019

Published online: 30 January 2019

References

1. Ghaffar S, Pearse RM, Gillies MA. ICU admission after surgery. Curr Opin Crit Care [Internet]. 2017;1. http://insights.ovid.com/crossref?an =00075 198-900000000-99242.

2. Guarracino F, Bertini P. To ICU or not to ICU: tailoring postoperative care in the face of reduced resources and increased morbidity. Minerva Anestesiol. 2017:83:134-5.

3. Niederman MS, Berger JT. The delivery of futile care is harmful to other patients. Crit Care Med. 2010;38:S518-22.

4. Knaus WA, Draper EA, Wagner DP, Zimmerman JE. APACHE Il: a severity of disease classification system. Crit Care Med [Internet]. 1985;13:818-29.

5. Moreno RP, Metnitz PGH, Almeida E, Jordan B, Bauer P, Campos RA, et al. SAPS 3-from evaluation of the patient to evaluation of the intensive care unit. Part 2: development of a prognostic model for hospital mortality at ICU admission. Intensive Care Med [Internet]. 2005;31:1345-55.

6. Vincent JL, Moreno R, Takala J, Willatts S, De Mendonça A, Bruining $\mathrm{H}$, et al. The SOFA (Sepsis-related Organ Failure Assessment) score to describe organ dysfunction/failure. Intensive Care Med. 1996;22:707-10.

7. Minne L, Abu-Hanna A, de Jonge E. Evaluation of SOFA-based models for predicting mortality in the ICU: a systematic review. Crit Care [Internet]. 2009;12:R161. https://doi.org/10.1186/cc7160.

8. Metnitz PG, Moreno RP, Almeida E, Jordan B, Bauer P, Campos RA, et al. SAPS 3-from evaluation of the patient to evaluation of the intensive care unit. Part 1: objectives, methods and cohort description. Intensive Care Med [Internet]. 2005;31:1336-44.

9. Sakr Y, Krauss C, Amaral ACKB, Réa-Neto A, Specht M, Reinhart K, et al. Comparison of the performance of SAPS II, SAPS 3, APACHE II, and their customized prognostic models in a surgical intensive care unit. $\mathrm{Br} J$ Anaesth. 2008;101:798-803.

10. Soares M, Salluh JIF. Validation of the SAPS 3 admission prognostic model in patients with cancer in need of intensive care. Intensive Care Med. 2006;32:1839-44

11. den Boer S, de Keizer NF, de Jonge E. Performance of prognostic models in critically ill cancer patients - a review. Crit Care [Internet]. 2005:9:458-63. 
12. Stephens RS, Whitman GJR. Postoperative critical care of the adult cardiac surgical patient. Part I: routine postoperative care. Crit Care Med. 2015;43:1477-97.

13. Vickers AJ, Elkin EB. Decision curve analysis: a novel method for evaluating prediction models. Med Decis Mak. 2006;26:565-74.

14. Vickers AJ. Decision analysis for the evaluation of diagnostic tests, prediction models, and molecular markers. Am Stat. 2008:62:314-20.

15. Allyn J, Ferdynus C, Bohrer M, Dalban C, Valance D, Allou N. Simplified acute physiology score II as predictor of mortality in intensive care units: a decision curve analysis. PLoS ONE. 2016;11:e0164828.

16. Yamamoto S, Yamazaki S, Shimizu T, Takeshima T, Fukuma S, Yamamoto Y, et al. Prognostic utility of serum CRP levels in combination with CURB-65 in patients with clinically suspected sepsis: a decision curve analysis. BMJ Open. 2015;5:e007049.

17. Cohen JF, Korevaar DA, Altman DG, Bruns DE, Gatsonis CA, Hooft L, et al. STARD 2015 guidelines for reporting diagnostic accuracy studies: explanation and elaboration. BMJ Open. 2016;6:e012799.

18. Moons KGM, Altman DG, Reitsma JB, loannidis JPA, Macaskill P, Steyenberg EW, et al. Transparent Reporting of a multivariable prediction model for Individual Prognosis or Diagnosis (TRIPOD): explanation and Elaboration. Ann Intern Med [Internet]. 2015;162:W1-74.

19. Vincent JL. Give your patient a fast hug (at least) once a day. Crit Care Med. 2005;33:1225-9.

20. Alba AC, Agoritsas T, Walsh M, Hanna S, lorio A, Devereaux PJ, et al. Discrimination and calibration of clinical prediction models. JAMA [Internet]. 2017;318:1377. https://doi.org/10.1001/jama.2017.12126.

21. DeLong E, DeLong D, Clarke-Pearson D. Comparing the areas under two or more correlated receiver operating characteristic curves: a nonparametric approach. JSTOR Biom. 1988;44(3):837-45.

22. Lemeshow S, Hosmer DWJ. A review of goodness of fit statistics for use in the development of logistic regression models. Am J Epidemiol [Internet]. 1982;115:92-106.
23. Vincent JL, Moreno R, Takala J, Willatts S, De Mendonca A, Bruining $\mathrm{H}$, et al. The SOFA (Sepsis-related Organ Failure Assessment) score to describe organ dysfunction/failure. On behalf of the Working Group on Sepsis-Related Problems of the European Society of Intensive Care Medicine. Intensive Care Med [Internet]. 1996;22:707-10.

24. Haniffa R, Isaam I, De Silva AP, Dondorp AM, De Keizer NF. Performance of critical care prognostic scoring systems in low and middle-income countries: a systematic review. Crit Care. 2018;22:18.

25. Salluh JIF, Soares M. ICU severity of illness scores. Curr Opin Crit Care [Internet]. 2014;20:557-65.

26. Vincent J-L, Moreno R, Moreno R, Moreno R, Jordan B, Metnitz P, et al. Clinical review: scoring systems in the critically ill. Crit Care [Internet]. 2010;14:207. https://doi.org/10.1186/cc8204.

27. Schenker Y, White DB, Crowley-Matoka M, Dohan D, Tiver GA, Arnold RM. "It hurts to know... and it helps": exploring how surrogates in the ICU cope with prognostic information. J Palliat Med. 2013;16:243-9.

28. Pearse RM, Rhodes A, Moreno R, Pelosi P, Spies C, Vallet B, et al. EuSOS: European surgical outcomes study. Eur J Anaesthesiol. 2011;28:454-6.

29. Pearse R, Moreno RP, Bauer P, Pelosi P, Metnitz P, Spies C, et al. Mortality after surgery in Europe: a 7 day cohort study. Lancet. 2012;380:1059-65.

30. Kahan BC, Koulenti D, Arvaniti K, Beavis V, Campbell D, Chan M, et al. Critical care admission following elective surgery was not associated with survival benefit: prospective analysis of data from 27 countries. Intensive Care Med. 2017;43:971-9.

31. Silva Junior JM, Malbouisson LMS, Nuevo HL, Barbosa LGT, Marubayashi LY, Teixeira IC, et al. Applicability of the simplified acute physiology score (SAPS 3) in Brazilian hospitals. Rev Bras Anestesiol. 2010;60:20-31.

\section{Submit your manuscript to a SpringerOpen ${ }^{\circ}$ journal and benefit from:}

- Convenient online submission

- Rigorous peer review

- Open access: articles freely available online

- High visibility within the field

- Retaining the copyright to your article

Submit your next manuscript at $\mathbf{s p r i n g e r o p e n . c o m ~}$ 